Revista Iberoamericana

de las Ciencias Sociales y

Humanísticas

ISSN: 2395 - 7972

https://doi.org/10.23913/ricsh.v10i19.248

Artículos Científicos

\title{
La asamblea comunal y su funcionamiento en el estado de Guerrero
}

\author{
The Communal Assembly and its Operation in the State of Guerrero
}

A assembleia comunal e seu funcionamento no estado de Guerrero

Silvina García Quezada

Universidad Autónoma de Guerrero, Facultad de Derecho, México

08015206@uagro.mx

https://orcid.org/0000-0002-2144-9638

Víctor Manuel Arcos Vélez

Universidad Autónoma de Guerrero, Facultad de Derecho, México

09442@uagro.mx

https://orcid.org/0000-0002-16412-6570

\section{Resumen}

El presente artículo expone cómo es que los pueblos originarios han fortalecido su identidad a través de la asamblea comunitaria, medio de justicia tradicional para solucionar los conflictos de índole local básico y para ejercer la toma de decisiones en la comunidad indígena, y que ha sido clave en el reconocimiento por primera vez en la historia de Guerrero de un pueblo con gobierno comunitario en Ayutla. Aplicando la investigación documental, la observación presencial de algunas asambleas comunales y analizando desde la antropología social los usos y costumbres de los pueblos originarios, se alcanzó a vislumbrar el desarrollo de medios de solución a conflictos tratados en las asambleas y cómo es que previo a estas se da un diálogo ciudadano ceremonial. Esta efectiva forma de organización está construyendo un sistema nuevo desde lo local. Se trata de una de las formas de organización campesina que tiene posibilidades de enraizarse y consolidarse como una fuerza de resistencia a la añeja política de destrucción del mundo indígena. 


\title{
Revista Iberoamericana
}

de las Ciencias Sociales y

Humanísticas

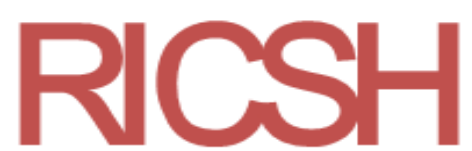

ISSN: $2395-7972$

Palabras clave: asamblea comunidad, convivencia comunal, gobernabilidad indígena, reconocimiento comunal.

\begin{abstract}
This article explains how indigenous peoples have strengthened their identity through the community assembly, a means of traditional justice to solve basic local conflicts and to exercise decision-making in the indigenous community, and that it has been key in the recognition for the first time in the history of Guerrero of a town with community government in Ayutla. Applying documentary research, face-to-face observation of some communal assemblies and analyzing from social anthropology the uses and customs of native peoples, it was possible to glimpse the development of means of solution to conflicts dealt with in the assemblies and how it is that prior to the treatment of conflicts there is a ceremonial citizen dialogue. This effective form of organization is building a new system from the local. It is one of the forms of peasant organization that has the potential to take root and consolidate itself as a force of resistance to the long-standing policy of destruction of the indigenous world.
\end{abstract}

Keywords: community assembly, communal coexistence, indigenous governance, communal recognition.

\section{Resumo}

Este artigo expõe como os povos indígenas fortaleceram sua identidade por meio da assembléia comunitária, um meio de justiça tradicional para resolver conflitos locais básicos e para exercer a tomada de decisões na comunidade indígena, e que isso foi fundamental para o reconhecimento pela primeira vez em a história de Guerrero de uma cidade com governo comunitário em Ayutla. Aplicando pesquisa documental, observação face a face de algumas assembléias comunais e analisando os usos e costumes dos povos indígenas a partir da antropologia social, foi possível vislumbrar o desenvolvimento de meios de solução para os conflitos tratados nas assembléias e como era isso antes disso, há um diálogo cerimonial com os cidadãos. Essa forma eficaz de organização está construindo um novo sistema local. É uma das formas de organização camponesa que tem potencial para se enraizar e se consolidar como força de resistência à política de destruição do mundo indígena de longa data. 


\section{Revista Iberoamericana}

de las Ciencias Sociales y

Humanísticas

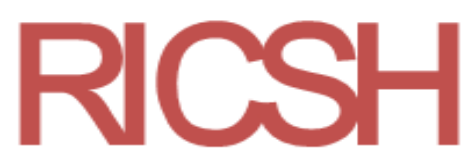

ISSN: $2395-7972$

Palavras-chave: assembleia comunitária, coexistência comunitária, governança indígena, reconhecimento comunitário.

Fecha Recepción: Mayo 2020

Fecha Aceptación: Diciembre 2020

\section{Introducción}

En el estado de Guerrero, ubicado en el extremo sur de México, una región que en las cuantificaciones de desarrollo de la nación aparece en el último lugar, se han asentado varias culturas indígenas a las orillas del río conocido en Puebla como Atoyac y en Guerrero como Mezcala-Balsas, así como Alto, Medio y Bajo Balsas, nominación aplicable solo al trayecto que atraviesa de oriente a poniente a toda la entidad federativa.

A la vera del río, los pueblos indígenas desarrollaron una serie de prácticas sociales, usos y costumbres que reivindican su derecho a tener una lengua (allí hablan una de las cuatro principales que existen en el estado), a tener control sobre su territorio y trabajar con autonomía. ¿Cómo asumen estos retos? Mediante asambleas comunitarias. En la casa del pueblo (la comisaría), los habitantes se reúnen habitualmente bajo convocatoria previa los días domingo para tomar las decisiones comunes más importantes. La asamblea es el medio por el cual emiten y escuchan con respeto las opiniones de cada uno de los integrantes, quienes participan exponen con toda libertad su acuerdo o desacuerdo, pero, en un momento dado, todos deben adherirse a una sola resolución, tiene que haber consenso.

\section{Objetivos}

Este trabajo tiene la intención de iniciar una serie de estudios sobre las formas de organización indígena; en Guerrero se presenta una muy particular. Se trata de un fenómeno estudiado por investigadores extranjeros, pero con pocos esfuerzos por investigadores nacionales, y casi no existen estudios locales y las organizaciones sociales, que son las más activas, casi siempre son demasiado subjetivas y politizadas en sus posiciones.

Por ello, nos hemos planteado presentar una serie de estudios sin inclinación ideológica: exponer solo hechos susceptibles de demostración analítica relacionados con el funcionamiento de la asamblea en las comunidades indígenas, y cómo se orienta esta hacia una forma de organización que está presentando resistencia a la política integracionista y de pérdida de la identidad indígena. Aun cuando en los textos legales se afirma que existe una política pública de reconocimiento de los saberes y culturas originarias, en el análisis de los 


\section{Revista Iberoamericana \\ de las Ciencias Sociales y Humanísticas}

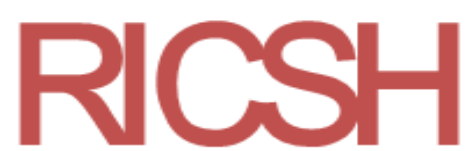

ISSN: $2395-7972$

textos legales encontramos que no existe una verdadera protección a la identidad comunitaria indígena, que son más bien posturas conservacionistas, de características muy similares a las reservaciones indígenas; se separa a aquellos que se niegan a insertarse en el sistema representativo de la organización político-partidista de los que reclaman el derecho a la autonomía y autodeterminación. Pese a todo, la realidad de los pueblos es cada vez más organizada y más consciente de su identidad cultural y su derecho a conservarla.

\section{Metodología}

En el presente artículo se ha aplicado la metodología de la investigación documental para revisar publicaciones anteriores sobre los grupos indígenas en la región guerrerense. Como ya lo hemos adelanto, los autores de estas publicaciones casi siempre son extranjeros y tienen una inclinación hacia la antropología social. También utilizamos el análisis sobre la organización de los pueblos originarios y aplicamos técnicas de observación directa de las asambleas comunales, así como conversaciones (entrevista a ciudadanos y comisariados) que giraron torno a cómo han desarrollado un sistema de organización como el caso de Ayutla de los Libres, Guerrero, en donde se reconoció al primer ayuntamiento comunal por usos y costumbres en este estado, acontecimiento que prevemos pronto se habrá de replicar en otros municipios más (igualmente es importante resaltar el activismo de la asociación Tlachinollan, en Tlapa, del grupo 500 años de Resistencia Indígena, de la Coordinadora Regional de Autoridades Comunitarias [CRAC] y de la Unión de Pueblos y Organizaciones del Estado de Guerrero [Upoeg], organizaciones que han encontrado férrea resistencia en contra de su funcionamiento por parte de grupos que se niegan a abandonar el ancestral control sobre comunidades indígenas).

\section{Resultados}

Hemos encontrado que se está generando un cambio en la organización de la sociedad rural en el estado de Guerrero, un cambio desde abajo, desde la población indígena, que se está organizando para que se reconozcan formas de gobierno comunitarias, tal y como en Ayutla. Esto solo se ha logrado con la organización indígena gestada en la asamblea comunitaria, cuyos resultados de gobernanza serán clave para que siga creciendo y se justifique como opción de integración del poder público en el ámbito local, al menos a lo 


\section{Revista Iberoamericana}

de las Ciencias Sociales y

Humanísticas

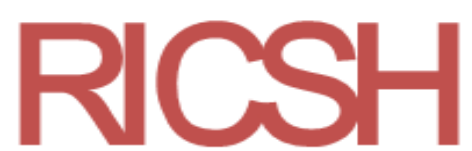

ISSN: 2395 - 7972

largo de los municipios en la zona sur-oriente, predominantemente indígenas, de los pueblos asentados originariamente a las márgenes del principal río guerrerense, el Balsas.

\section{Discusión}

Guerrero, México, cuenta con $64281 \mathrm{~km}^{2}, 3.2 \%$ de la superficie total del país, y alberga a 3500000 habitantes, de los cuales 700000 son indígenas (17.2\% de la población total indígena en el país) (Gobierno del Estado de Guerrero, 2016). Ubicado en el extremo sur del país, su geografía agreste es consecuencia de la formación de una cadena montañosa, cuyas cimas superan los 3000 m s. n. m., conocida como el filo mayor de la Sierra Madre del Sur, que termina al oriente en la región mixteca. Este nombre se le da porque las aguas que la atraviesan corren del filo al sur, a la plataforma continental que forma la costa, y del otro lado desciende el agua hacia el norte, en la depresión del Balsas, río que atraviesa todo el estado, de oriente en diagonal descendente a poniente, derivando al sur, y que tiene una longitud de $701 \mathrm{~km}$.

En su curso atraviesa cuatro estados distintos: Guerrero, Michoacán, y Tlaxcala y Puebla. (...) De la misma forma, establece contacto con otros estados del país, puesto que drena por Veracruz, Morelos, Oaxaca, México y Jalisco.

En la región mixteca, que es la parte oriental descendiendo al sur, encontramos a Ayutla de los Libres, en donde los pueblos tlapaneco (Me-pha) y mixteco (Tu'un-savi), aun con la oposición de los habitantes mestizos, tras la experiencia fallida de Tecoanapa, lograron en una elección constitucional local configurar un gobierno municipal comunitario por primera vez en la historia de Guerrero.

La región de Ayutla ha sido zona de influencia de culturas precolombinas como el pueblo mixteco; tu'un savi, que habita en la región; ñu'un nuu savi ('país de la lluvia'), conformada por los municipios de Alcozauca, Atlamajalcingo, Ayutla, Copanatoyac, Igualapa, Metlatónoc, San Luis Acatlán, Tlacoachistlahuaca y Xalpatlahuac (Mindek, 2003), que coinciden geográficamente con sus vecinos del pueblo tlapaneco, así llamados por los nahuas; mbo me phaa ('habitante de Tlapa'), que ocupan los municipios de Tlapa, Atlixtac, Malinaltepec, Tlacoapa, Zapotitlán Tablas (Serrano, 2006), y cuyas aguas descienden a la región ocupada por los pueblos nahuas, Apango (Mártir de Cuilapan), Copalillo, Atenango del Río, Tepecoacuilco, Apaxtla, Cocula, Teloloapan y Zumpango del Rio (Good y Barrientos, 2004), descendientes del pueblo mexica o azteca. 


\section{Revista Iberoamericana de las Ciencias Sociales y Humanísticas}

ISSN: $2395-7972$

Es por ello por lo que en esta región sur del país aún se conservan amplias zonas rurales y al menos en 10 de estos municipios existe predominancia de habitantes indígenas. Por supuesto, en la región hay una diversidad de lenguas y tradiciones, aunque también existe un factor común, los llamados usos y costumbres. Y entre algunos de estos pueblos persisten conflictos, como el de Alacatlatzala con Atlamajalcingo (Velázquez, 2017), pues las autoridades municipales pretenden su integración, su participación y su apertura al sistema jurídico aplicado en nuestro territorio nacional, pero ellos se resisten afirmando que tienen derecho a la autodeterminación en términos del artículo 2 constitucional y el Convenio 169 de la Organización Internacional del Trabajo [OIT] (24 de enero de 1991).

Esto se hace igualmente evidente cuando vemos que la Constitución Política del Estado Libre y Soberano de Guerrero, en su artículo 13, dispone que cada población debe nombrar a un representante del gobierno municipal, que recibe el nombramiento de comisario municipal.

El Gobierno del Estado en coordinación con las autoridades municipales (...) generará el cúmulo de políticas públicas que promuevan el acceso a los derechos humanos y la igualdad de oportunidades a los pueblos indígenas y comunidades afromexicanas (...). Las obligaciones de cada uno de los poderes del Estado se determinarán en una ley reglamentaria $(H$. Congreso del Estado de Guerrero, 2014, p. 8).

Ampliando la aplicación de los principios constitucionales, la Ley Orgánica del Municipio Libre del Estado de Guerrero (Gobierno del Estado de Guerrero, 18 de febrero 2020), vigente desde 1990 y actualizada en 2020, establece, a lo largo de varios artículos, los siguientes puntos:

- Los municipios que integran al estado se administrarán por ayuntamientos de elección popular directa (art. 5).

- Los municipios se dividirán en comisarías municipales, las que contarán con un mínimo de 1000 habitantes e infraestructura para las funciones (art. 16).

- Las comisarías son órganos de desconcentración territorial en la administración pública municipal, a cargo de un comisario electo en votación popular directa por medio de mecanismos vecinales a través del sufragio (...) y que tendrán el carácter honorífico (art. 34).

- La administración de las comisarías estará a cargo de un comisario propietario, de un comisario suplente y de dos vocales (art. 81). 


\section{Revista Iberoamericana}

de las Ciencias Sociales y

Humanísticas

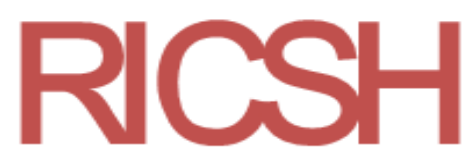

ISSN: $2395-7972$

El primer año actuará la planilla completa; el segundo año cesará en sus funciones el comisario, y asumirá ese carácter el primer comisario vocal, pasando el suplente a fungir como segundo comisario vocal, y este a primer comisario vocal. El tercer año, el Segundo comisario vocal actuará como comisario, y el suplente como primer comisario vocal

En las poblaciones que se reconozcan como indígenas, los comisarios municipales o delegados se elegirá un propietario y un suplente en la segunda quincena del mes de diciembre de cada año mediante el método de sus usos y costumbres, mismos que deberán tomar protesta ante la autoridad municipal en la primera quincena del mes de enero, quienes durarán por el periodo de un año (p. 81).

Pero, como está sucediendo en casi todas las regiones del país, la inseguridad, la afectación a la vida y al patrimonio de las familias se ha incrementado por el cultivo y trasiego de productos ilícitos como los estupefacientes, la minería y la explotación maderera por concesiones federales, que permiten la explotación irracional de los recursos naturales, principalmente en manos de extranjeros a través de los mestizos y sus trabajadores, a los que las comunidades indígenas les llaman ladinos, pues la mayoría son más indígena que blancos (afirman), pero se afrentan de que los llamen indios. Estas particulares circunstancias han derivado en el crecimiento acelerado de la delincuencia, a tal grado que los pueblos se han visto obligados a enfrentarlos con armas para protegerse a sí mismo (Matias, 2014).

La respuesta a estas presiones ha sido la organización comunitaria a través de las asambleas.

Existen dos tipos de asamblea. En primer lugar, la derivada de dar cumplimiento a las obligaciones del pueblo con la autoridad municipal, acompañando a su comisario, para el cumplimiento de los compromisos municipales.

El comisario municipal, un cargo honorífico reconocido por la autoridad del municipio, con duración de un año y continuado por la lista de suplentes, cada uno un año en su encargo hasta completar los tres años una para nueva elección; este cargo es el enlace entre la comunidad y el "Gobierno", al cual se le asignan el control de la policía comunitaria y la operación del registro civil, los caminos, las obras y servicios básicos dentro de la comunidad y sus caseríos anexos.

En segundo lugar, está la asamblea agraria, que involucra de manera específica a los titulares de los derechos de propiedad sobre la tierra, los comunitarios. Debe ser convocada 


\section{Revista Iberoamericana}

de las Ciencias Sociales y

Humanísticas

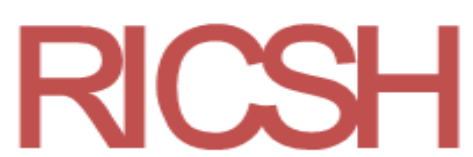

ISSN: 2395 - 7972

previa convocatoria acordada, con las autoridades agrarias y presidida por el comisariado ejidal, que es un órgano colegiado que decide el destino del uso del suelo, para habitación, para cultivo, para el manejo del ganado y para las relaciones con sus pares en las comunidades vecinas, y marca las políticas de organización que la comunidad debe establecer en su centro de población y caseríos anexos.

Pero, en la realidad, ambas autoridades, tanto la civil como la agraria, usualmente acuerdan los asuntos y participan en la asamblea comunal. Por ello las reuniones son generales y no separadas.

\section{De las formas de la asamblea comunal}

Así pues, en las asambleas generales participan ambos comisarios y pueden distinguirse varios tipos de asamblea: las ordinarias, las extraordinarias y las de alarma.

a) Las reuniones ordinarias se realizan en la comisaría los días domingo y tratan asuntos como la elección de autoridades, la autorización para la celebración de la fiesta patronal, fajinas para reparación del camino, la parcela escolar, solicitar maestros, solicitar enfermera para el centro de salud, la seguridad pública en la comunidad y sus anexos. Cabe señalar que, respecto a esta última, se organiza mediante el nombramiento de policías comunitarios y sus comandantes, un cargo honorífico pero obligatorio, quienes están credencializados para que puedan portar armas, generalmente escopetas y rifles de bajo calibre, aunque en sus domicilios tienen armas de alto poder que usan solo en caso de emergencia, a sabiendas de que en ambos casos entran en conflicto con las autoridades de los tres niveles de Gobierno, pero es un cargo que no pueden renunciar ni negarse a prestar su servicio, y que cuando termine su periodo otros los relevarán.

b) Las reuniones extraordinarias "son convocadas a petición de las autoridades ante temas específicos o coyunturas críticas" (Abogados-Puebla, 2020), por ejemplo, la violación a los linderos de la tierras, conflictos de extracción de materiales o productos sin el consentimiento del comisariado, o todas aquellas que requieran de atención de urgente resolución.

c) Las de alarma son aquellas que requieren de la reunión del pueblo y que generalmente son convocadas por el repicar de campanas, que anuncia tragedias acaecidas o inminentes como incendios, inundaciones, hechos ilícitos que han provocado el daño de algún miembro de la comunidad o detención y aseguramiento de personas, o casos graves que requieren una atención y participación inmediata de comuneros y avecindados, en cuyo 


\section{Revista Iberoamericana \\ de las Ciencias Sociales y Humanísticas}

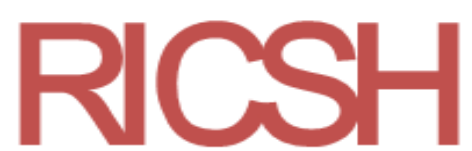

ISSN: 2395 - 7972

caso quedan en sesión permanente y la comisaría es el lugar a donde todos se concentran hasta que las autoridades declaren terminada la emergencia.

En estos casos se tiene un libro de actas, que custodian celosamente, y de todo evento o asamblea se registra lo acontecido con mucho detalle. Al respecto, toda acta de asamblea debe contener:

a) El número de convocatoria emitida (primera, segunda o tercera).

b) La lista de asistencia.

c) La verificación del quorum legal.

d) La declaración de instalación de la asamblea.

e) El nombramiento de la mesa de debates (presidente, secretario y escrutadores).

f) Los asuntos a desahogar y los acuerdos tomados en cada uno de ellos.

g) Las tareas y medidas para ejecutar los acuerdos.

h) La clausura de la asamblea.

i) Las firmas y sellos del comisariado, consejo de vigilancia y mesa de debates.

Para las asambleas se toma como fundamento la Ley Agraria (Presidencia de la República, 25 de junio de 2018), vigente desde 1992 y cuya última reforma fue realizada en el 2018, en la cual se establece que los núcleos de población tienen personalidad jurídica propia, cuentan con patrimonio y los ejidos y comunidades pueden contar con reglamentación interna, que pueden estar conformadas por los titulares de los derechos agrarios y por avecindados. A este respaldo jurídico habría que añadir la Ley 701 y el Convenio 169 de la OIT.

La Ley Agraria, en su artículo 21, dice que son órganos de los ejidos y comunidades la asamblea, el comisariado y el consejo de vigilancia; también, que las resoluciones de la asamblea se tomarán por mayoría de votos, pero estas serán obligatorias para todos los ausentes y disidentes a la asamblea. De toda asamblea, se levantará acta circunstanciada, la cual será firmada por el comisariado y el consejo de vigilancia, que no requieren de validación y sus acuerdos serán inscribibles en el Registro Agrario Nacional con la misma fuerza que un documento con fe pública y servirá para acreditar los derechos agrarios a favor de quien se emite, así como sus modificaciones en su caso (Presidencia de la República, 25 de junio de 2018).

La Ley Agraria establece un capítulo específico para las comunidades (de los artículos 98 al 107), el cual deja en libertad a los comunitarios con derechos agrarios acreditados para poder cambiar de régimen, bien de comunal a ejidal o viceversa, aun cuando hemos 


\section{Revista Iberoamericana \\ de las Ciencias Sociales y Humanísticas}

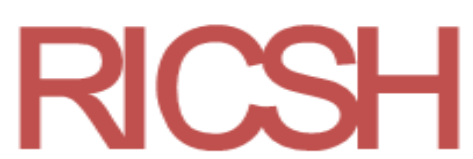

ISSN: 2395 - 7972

observado que la forma jurídica preferida por las comunidades indígenas es la comunitaria. Entonces, el nombre de comunidad deviene del régimen de propiedad en su origen, pero se asume como una forma de organización social característica de los grupos étnicos de ascendencia indígena.

\section{La democracia en la comunidad indígena}

Podemos hablar de la democracia comunal desde hace muchos años. La fuerza campesina e indígena es la que facilitó la independencia, la reforma y la revolución. Sin embargo, su estudio formal como una alternativa a la dinámica de organización de la sociedad se ha venido documentando desde la década de los años 60, cuando comienza a describirse como se ha venido desarrollando: un proceso político orientado a la construcción de un sistema democrático, representativo. Inicialmente este proceso se concentraba en el partido político dominante; en aquellos años, mediante una organización que conformaba uno de sus tres sectores, el sector campesino con la Confederación Nacional Campesina (CNC), toda vez que aún no existía oposición para la alternancia.

El debilitamiento del régimen del partido dominante a partir de los comicios de 1988, y el surgimiento de una oposición, dio fuerza a los movimientos sociales para su creciente participación, que reflejados en los procesos de conformación de organizaciones disidentes permitieron la creación de un movimiento indígena, proceso que culmina con el levantamiento zapatista en la zona indígena chiapaneca, que rescató fundamentalmente la experiencia comunal de las organizaciones indígenas y la fortalecieron ideológicamente con el lenguaje precedente del derecho internacional, principalmente el convenio 169 de la OIT, que influyó en las políticas públicas para reconocer el derecho a la conciencia de identidad indígena, de manera genérica establecida en el artículo 2 de la constitución mexicana.

Al respecto, Pablo González Casanova (2009) comentaba lo siguiente:

Las teorías acerca de la democracia con justicia social y dignidad, de la "democracia de todos", como la llaman los zapatistas, incluyen planteamientos científicos y humanísticos superiores a los de cualquier otra teoría sobre la organización del poder y la sociedad en las ciudades, los pueblos, las naciones y el mundo.

(...) Hacerlo va más allá del legado y la perspectiva de las ciencias sociales, hacia la construcción y creación, en la teoría y la realidad, de un nuevo paradigma histórico de democracia universal no excluyente, con 


\section{Revista Iberoamericana \\ de las Ciencias Sociales y Humanísticas}

ISSN: 2395 - 7972

connotaciones morales y prácticas, humanistas y científicas, utópicas y políticas; con reestructuraciones de los intereses particulares y de los intereses generales.

(...) En sus contingentes no sólo se encuentran los herederos de una lucha de resistencia que dura más de 500 años, sino quienes vienen de los movimientos más recientes del pensamiento revolucionario y de la teología de la liberación. En todos ellos se advierten las experiencias mexicanas del pensar liberador y democrático (pp. 219 y 239).

Referente a este asunto, de cómo puede participar el pueblo en el avance democrático de las instituciones, encontramos que la verdadera participación del pueblo se da en los espacios geográficos menores, entendiendo que estos espacios geográficos son las comunidades, pues es en ellos donde las familias se reconocen entre sí y pueden relacionarse más fácilmente, y que pueden reunirse sin depender de algún medio de transporte, porque son vecinos cercanos y comparten problemas similares, de tipo económico, social, educativos, de comunicación, de seguridad y los más diversos temas que para ellos les resulten de verdadera importancia.

A partir de ese diálogo dentro de la asamblea nace la verdadera democracia, pues es ahí donde los asuntos se discuten y resuelven; y no siempre resulta fácil llegar a la toma de decisiones, los asistentes gozan del derecho de poder expresar su visión del asunto que se trata, argumentan y escuchan a quienes están en desacuerdo, pero al final quien ha de tomar la decisión final es la asamblea.

La asamblea, por tanto, es un espacio de discusión y deliberación, de organización y toma de decisiones.

En América Latina el término comunidad puede asociarse (sin que esto signifique impugnar las innumerables acepciones alternativas) con prácticas económicas y culturales que encuentran sus raíces en los pueblos indígenas.

(...) En su enfrentamiento con corporaciones y gobiernos, los pueblos campesinos e indígenas suelen enfatizar su derecho a determinar por sí mismos qué tipo de desarrollo económico quieren que se promueva en sus territorios, entendidos estos últimos como espacios para la reproducción de la vida. Resulta interesante percatarse de que esto supone una reelaboración de lo "público" que distingue la esfera de lo estatal, lo privado y lo "comunitario" (p. 66 y 76-77). 


\section{Revista Iberoamericana}

de las Ciencias Sociales y

Humanísticas

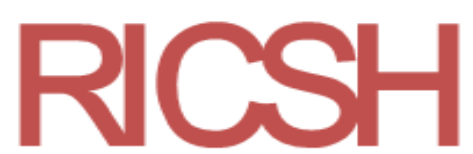

ISSN: $2395-7972$

¿Por qué es una organización? Porque la comunidad, similar a las polis griegas, está integrada por individuos que se identifican, tienen sentido de pertenencia y empoderamiento social, y en el momento en que se reúnen dejan de tener intereses individuales y se transforman en partes de un cuerpo social con un interés superior: la comunidad.

La organización comunitaria es importante porque es ahí donde damos a conocer nuestros valores humanos y talentos individuales para resolver de forma efectiva los problemas sociales, económicos, productivos, ambientales y políticos de la comunidad. Mujeres y hombres como seres sociales que somos, necesitamos de la organización con nuestros semejantes para comunicarnos, socializarnos, desarrollarnos integralmente y buscar condiciones más justas para la sociedad en la cual vivimos (Rodríguez, s. f., párr. 2).

Las comunidades han aprendido a tener mayor contacto con organizaciones no gubernamentales (ONG), o con organismos internacionales interesadas en su cultura, lengua o tradiciones, ya sean nacionales o extranjeras, por eso no es extraño ver a mochileros que se mueven entre el gentío, muchos de ellos revolucionarios que vienen a predicar lo que en sus países de origen no pueden hacer.

En consecuencia, la asamblea comunitaria indígena funciona en una dinámica de democracia comunal, que se puede manifestar de las siguientes formas:

a) Es un sistema patriarcal, en parte, pues quienes deciden son los "principales", puesto que se ganan al interior como consecuencia de un sistema tradicional de cargos y jerarquía cívico-religiosa, a través de las llamadas "mayordomías".

b) En la asamblea se escucha a los hombres aun cuando las mujeres asisten (siempre que sean solas), y tienen la libertad de elegir de forma directa a las autoridades, más bien avalar la decisión tomada previamente por los principales (recuérdese que es un órgano legitimador) y de esa forma organizan sus funciones e imponen límites, así como obligaciones de rendir cuentas, en la que todos se sienten participantes.

c) La función de la autoridad es la de interpretar la voluntad general, verbalizada, que implica el sentimiento de la comunidad, muchas veces inducida, pero convencidos de que fueron ellos quienes deciden. Las participaciones en la asamblea los convencen de que no se trata de escoger una de varias opciones posibles y desechar las demás, sino que todos coincidan en una sola opción que integra a todas las opiniones. 


\section{Revista Iberoamericana de las Ciencias Sociales y Humanísticas}

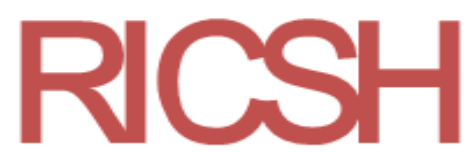

ISSN: 2395 - 7972

d) Por lo tanto, el poder reside en la asamblea de los ciudadanos y ciudadanas por las personas que habitan en esa comunidad, convencidos de que todos eligieron a las personas para que formen el consejo comunal. Es la máxima autoridad de la comunidad y sus decisiones tienen poder vinculante para el consejo comunal, ya que trasladaron su poder individual en un poder de la asamblea comunal.

La asamblea dejó de ser la reunión de los abuelos de antaño; ahora son los jóvenes quienes empujan la organización y defensa de sus territorios, de sus aguas, de su cultura, por ello, durante sus sesiones, asumen un poder absoluto, cuasiconstituyente, que puede respetar los acuerdos anteriores o establecer nuevos acuerdos desconociendo los anteriores, según se convenza a la asamblea.

Una asamblea comunal es, entonces, una aspiración a una forma de autogobierno de una pequeña colectividad, la cual se conforma como una instancia democrática en donde se discuten y toman decisiones para solucionar las problemáticas existentes con la finalidad de construir una nueva sociedad, pero que, de ninguna manera, sustituye a la figura del Estado, pues su alcance, aun cuando el ya mencionado artículo 2 constitucional permite la "libre determinación" (Cámara de Diputados del H. Congreso de la Unión, 11 de marzo de 2021), no está del todo vislumbrado.

Solo que hay un problema. Cada comunidad llega a sus acuerdos de forma independiente a las demás; son reacios a las leyes de los "ladinos" que rigen los municipios, por lo que pierden, al final, lo que pretendieron lograr: una democracia participativa, que sea respetada por los demás, y así sus autoridades solo alcanzan a convertirse en negociadores con sus pares en las etnias o con los ladinos que establecieron otros sistemas de designación de autoridades a través de los partidos políticos, pero en la actualidad solo en Ayutla de los Libres han logrado establecer un gobierno comunitario por usos y costumbres, es su primer logro, pero todavía no se consolida como una opción de gobierno.

Las cosas y los acontecimientos que en este estudio hemos encontrado son muy importantes en todo sentido, porque no todas las personas conocen este tema fuera del núcleo comunal, lo que ocurre es que la población no indígena en estos días no les parece interesante este tipo de situaciones. Anteriormente, en la comunidad indígena, cuando sucedía un conflicto, los habitantes solo esperaban a que la policía municipal de los ladinos llegara para hacerse cargo, la cual, al no encontrarse cerca de las comunidades indígenas, tardaba en acudir al lugar del conflicto; estas acciones tardías terminaban en conflictos aún más grandes debido a la venganza de los familiares de las víctimas. 


\section{Revista Iberoamericana}

de las Ciencias Sociales y

Humanísticas

ISSN: $2395-7972$

En un ejemplo solo como referencia, en una ocasión decía un ciudadano que entre su familia peleaban un terreno que habían heredado de sus abuelos, por lo cual discutían todo el tiempo sin llegar a un acuerdo, derivado de estos roces uno de ellos se molestó y con un machete laceró el brazo de su hermano.

Esto provocó la ruptura de la paz en la comunidad. La policía municipal que acudió, en lugar de solucionar los problemas, los complicaron. Por eso, ahora algunas comunidades tienen a sus llamados policías comunitarios, quienes son los que resguardan el orden para evitar este tipo de problemas y han tomado el papel que deberían realizar los policías municipales, pero es demasiado poco lo que se invierte en seguridad y es tan fuerte el miedo de las autoridades que no permiten que los ciudadanos se armen para defenderse. Sin embargo, en la práctica ahora Guerrero está lleno policías ciudadanos armados en las comunidades indígenas, y también se han armado en los pueblos y en las cabeceras municipales, que han sustituido a la seguridad policiaca municipal.

Por eso es importante ver el funcionamiento de las asambleas comunales. Escuchar de voz de los propios integrantes cuál es su forma de participación y modo de intervención dentro del desarrollo de una asamblea comunitaria indígena con la finalidad de entender de forma especial su modo de gobernabilidad autónoma.

\section{Conclusiones}

Las comunidades son los espacios en donde se genera la democracia de abajo hacia arriba. A través de las asambleas, los pueblos y comunidades predominantemente indígenas han encontrado la génesis para el gobierno comunitario, mediante el cual se vinculan los ciudadanos de forma directa, otorgando mandato a los representantes del pueblo y las convierten en autoridades.

Las asambleas son un medio de expresión de democracia directa a mano alzada, en donde los acuerdos son legítimos en sí mismos y merecedores de la mayor confianza de que son manifestaciones de la voluntad común, y no la imposición autoritaria de alguien con poder que lo decide o manda sin tomar en cuenta el sentimiento comunal.

Por su parte, los gobiernos municipales ahora tendrán un competidor para gobernar los municipios. Si la administración comunitaria resulta tener mejores resultados, entonces esto se extenderá a otros espacios. Por ello la oposición férrea de los partidos políticos contrarios, y sus mecanismos de control tradicionales, que se niegan a la asamblea comunal y su característica toma de decisiones. Una u otra determinarán el futuro de cómo gobernar 


\section{Revista Iberoamericana}

de las Ciencias Sociales y Humanísticas

ISSN: $2395-7972$

a los espacios comunales, al menos en esta zona sur oriente, en donde las culturas originarias están despertando y reclamando el derecho de autodeterminarse.

Este trabajo no tiene la intención de incidir sobre las formas de organización indígena en asambleas comunales, sino solo de narrar lo que está ocurriendo. La forma de organización y poder municipal está en manos de esas dos opciones: la forma tradicional de gobierno por partidos o la forma de gobierno comunal. Los resultados de sus administraciones seguramente lo determinarán.

\section{Futuras líneas de investigación}

Los integrantes del grupo de trabajo del cuerpo académico en consolidación 141, "Sistemas de Justicia en México", de la Facultad de Derecho de la Universidad Autónoma de Guerrero, estamos desarrollando investigaciones sobre derechos indígenas y formas de organización comunal. Y en conjunto hemos planeado un trabajo de investigación sobre la elección del municipio comunitario de Ayutla de los Libres en junio de 2021. También tenemos presentada una tesis de licenciatura sobre un análisis jurídico y político del gobierno comunal de Cherán, una tesis de maestría sobre gobierno indígena, un libro publicado por una editorial nacional, así como otros trabajos en proceso que estarán trabajando docentes y estudiantes de licenciatura y maestría en derecho, con lo que pretendemos ampliar el cúmulo de estudios locales sobre derecho y organización indígena en Guerrero y Michoacán, y contribuir a formar recursos humanos de alta calidad en el programa de posgrados de calidad del Consejo Nacional de Ciencia y Tecnología (Conacyt) como parte de nuestra funciones sustantivas en el trabajo universitario. 


\section{Revista Iberoamericana}

de las Ciencias Sociales y

Humanísticas

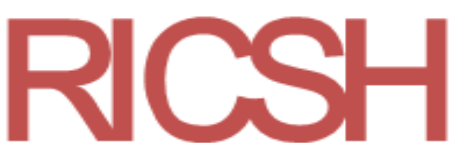

ISSN: $2395-7972$

\section{Referencias}

Abogados Puebla. (s. f.). Diferencia entre asambleas ordinarias y extraordinarias. Abogados

Puebla. Recuperado de http://abogadospuebla.com/diferencia-entre-asambleasordinarias-y-extraordinarias/.

AN / OC. (3 de octubre de 2020). ¿Cómo son las asambleas comunitarias en México?: Las Plumas de la Serpiente | Video. Aristegui Noticias. Recuperado de https://aristeguinoticias.com/0409/kiosko/como-son-las-asambleas-comunitarias-enmexico-las-plumas-de-la-serpiente-video/.

Cámara de Diputados del H. Congreso de la Unión. (11 de marzo de 2021). Constitución Política de los Estados Unidos Mexicanos. Diario Oficial de la Federación. Recuperado de http://www.diputados.gob.mx/LeyesBiblio/pdf/1_110321.pdf.

Cámara, F. (1996). Organización religiosa y política en Mesoamérica. En Korsbaek, L., Introducción al sistema de cargos (pp. 113-160). Toluca, México: Universidad Autónoma del Estado de México.

Cruz, G. (2016). Asamblea Comunal. México | Enciclopedia Jurídica Online. Recuperado de https://mexico.leyderecho.org/asamblea-comunal/.

Gobierno del Estado de Guerrero. (2016). Plan Estatal de Desarrollo 2016-2021. Guerrero, México: Gobierno del Estado de Guerrero. Recuperado de http://transparencia.guerrero.gob.mx/files/2016/04/Plan-Estatal-de-Desarrollo-20162021.pdf.

Gobierno del Estado de Guerrero. (18 de febrero 2020). Ley Orgánica del Municipio Libre del Estado de Guerrero. Periódico Oficial del Gobierno del Estado, (14). Recuperado de http://i.guerrero.gob.mx/uploads/2020/10/1-Ley-Org-Mpio-Libre.pdf.

González, P. (1965). La democracia en México. México: Era.

González, P. (2009). De la sociología del poder a la sociología de la explotación: pensar América Latina en el siglo XXI. México: Siglo del Hombre Editores y Clacso.

Good, C. y Barrientos, G. (2004). Nahuas del Alto Balsas. Ciudad de México, México: Comisión Nacional para el Desarrollo de los Pueblos Indígenas, Programa de las Naciones Unidas para el Desarrollo. Recuperado de http://www.cdi.gob.mx/dmdocuments/nahuas_alto_balsas.pdf.

H. Congreso del Estado de Guerrero. (20 de junio de 2020). Constitución Política del Estado Libre y Soberano de Guerrero. Periódico Oficial del Gobierno del Estado de Guerrero, (50). Recuperado de http://congresogro.gob.mx/62/legislacion/. 


\section{Revista Iberoamericana}

de las Ciencias Sociales y Humanísticas

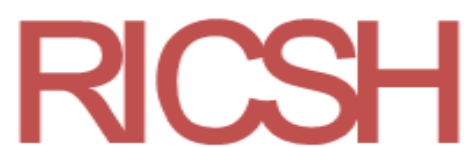

ISSN: $2395-7972$

Liceaga, G. (2013). El concepto de comunidad en las ciencias sociales latinoamericanas: apuntes para su comprensión. Cuadernos Americanos, (145), 57-85. Recuperado de http://www.cialc.unam.mx/cuadamer/textos/ca145-57.pdf.

Maldonado, K. y Aquino, A. (30 de junio de 1999). Organización colectiva y lucha comunitaria en Chiapas. La Jornada.

Matías, M., Aréstegui, R. y Vázquez, A. (2014). La rebelión ciudadana y la justicia comunitaria en Guerrero. México: Centro de Estudios Sociales y de Opinión Pública, Cámara de Diputados / LXII Legislatura.

Mendez, A. (2019). Río Balsas: ubicación, ¿dónde nace?, contaminación y mucho más. Conoce los ríos. Recuperado de https://conocelosrios.com/c-mexico/rio-balsas/.

Mendoza, A. (4 de junio de 2018). Para entender a la policía comunitaria de Guerrero. Nexos. Recuperado de https://seguridad.nexos.com.mx/?p=746.

Mindek, D. (2003). Mixtecos. México: Comisión Nacional para el Desarrollo de los Pueblos Indígenas.

Recuperado

de https://www.gob.mx/cms/uploads/attachment/file/11727/mixtecos.pdf.

Organización Internacional del Trabajo [OIT]. (24 de enero de 1991). Convenio 169 sobre pueblos indígenas y tribales. Diario Oficial de la Federación. Recuperado de https://www.senado.gob.mx/comisiones/desarrollo_social/docs/marco/Convenio_16 9_PI.pdf.

Presidencia de la República. (25 de junio de 2018). Ley Agraria. Diario Oficial de la Federación.

Recuperado

de http://www.diputados.gob.mx/LeyesBiblio/pdf/13_250618.pdf.

Rodríguez, A. (s. f.). Organización comunitaria: ¿Cuál es su importancia para el desarrollo humano y qué impactos genera? Emprende 3.0. Recuperado de http://emprend3.com/organizacion-comunitaria-cual-es-su-importancia-para-eldesarrollo-humano-y-que-impactos-genera/.

Serrano, E. (2006). Regiones indígenas de México. Ciudad de México, México: Comisión Nacional para el Desarrollo de los Pueblos Indígenas, Programa de las Naciones Unidas para el Desarrollo. Recuperado de http://www.cdi.gob.mx/regiones/regiones_indigenas_cdi.pdf.

Velázquez, L. (2017). Historia de los conflictos agrarios en Atlamajalcingo del Monte, Guerrero. (Tesis de maestría). Universidad Autónoma de Guerrero, Chilpancingo de los Bravo. Recuperado de http://ri.uagro.mx/handle/uagro/87. 


\section{Revista Iberoamericana de las Ciencias Sociales y Humanísticas}

\begin{tabular}{|c|c|}
\hline Rol de Contribución & Autor (es) \\
\hline Conceptualización & $\begin{array}{l}\text { García Quezada Silvina (Principal) y Arcos Vélez Víctor } \\
\text { Manuel (Principal) }\end{array}$ \\
\hline Metodología & $\begin{array}{l}\text { Arcos Vélez Víctor Manuel (Principal) García Quezada } \\
\text { Silvina (Apoyo) }\end{array}$ \\
\hline Software & No Aplica. \\
\hline Validación & $\begin{array}{l}\text { Arcos Vélez Víctor Manuel (Principal) García Quezada } \\
\text { Silvina (Apoyo) }\end{array}$ \\
\hline Análisis Formal & $\begin{array}{l}\text { García Quezada Silvina (Principal) y Arcos Vélez Víctor } \\
\text { Manuel (Principal) }\end{array}$ \\
\hline Investigación & $\begin{array}{l}\text { García Quezada Silvina (Principal) y Arcos Vélez Víctor } \\
\text { Manuel (Principal) }\end{array}$ \\
\hline Recursos & $\begin{array}{l}\text { García Quezada Silvina (Principal) y Arcos Vélez Víctor } \\
\text { Manuel (Principal) }\end{array}$ \\
\hline Curación de datos & $\begin{array}{l}\text { García Quezada Silvina (Principal) y Arcos Vélez Víctor } \\
\text { Manuel (Principal) }\end{array}$ \\
\hline $\begin{array}{l}\text { Escritura - Preparación del } \\
\text { borrador original }\end{array}$ & $\begin{array}{l}\text { García Quezada Silvina (Principal) y Arcos Vélez Víctor } \\
\text { Manuel (Principal) }\end{array}$ \\
\hline $\begin{array}{l}\text { Escritura - Revisión y } \\
\text { edición }\end{array}$ & $\begin{array}{l}\text { Arcos Vélez Víctor Manuel (Principal) García Quezada } \\
\text { Silvina (Apoyo) }\end{array}$ \\
\hline Visualización & $\begin{array}{l}\text { García Quezada Silvina (Principal) y Arcos Vélez Víctor } \\
\text { Manuel (Principal) }\end{array}$ \\
\hline Supervisión & $\begin{array}{l}\text { Arcos Vélez Víctor Manuel (Principal) García Quezada } \\
\text { Silvina (Apoyo) }\end{array}$ \\
\hline Administración de Proyectos & $\begin{array}{l}\text { Arcos Vélez Víctor Manuel (Principal) García Quezada } \\
\text { Silvina (Apoyo) }\end{array}$ \\
\hline Adquisición de fondos & $\begin{array}{l}\text { Arcos Vélez Víctor Manuel (Principal) García Quezada } \\
\text { Silvina (Apoyo) }\end{array}$ \\
\hline
\end{tabular}

